\title{
Mechanisms of Ultrasonic Modulation of Multiply Scattered Coherent Light: An Analytic Model
}

\author{
Lihong V. Wang* \\ Optical Imaging Laboratory, Biomedical Engineering Program, Texas A\&M University, \\ 3120 TAMU, College Station, Texas 77843-3120 \\ (Received 19 January 2001; published 10 July 2001)
}

\begin{abstract}
An analytic model of the ultrasonic modulation of multiply scattered coherent light in scattering media is developed based on two mechanisms: the ultrasonic modulation of the index of refraction and the ultrasonic modulation of the displacements of Rayleigh scatterers. In water solutions, for example, the first mechanism is slightly less important than the second mechanism when the scattering mean free path is less than a critical fraction (0.0890) of the acoustic wavelength, and it becomes increasingly more important beyond this point. This model agrees well with an independent Monte Carlo model.
\end{abstract}

DOI: 10.1103/PhysRevLett.87.043903

PACS numbers: 42.25.Dd, 42.90. $+\mathrm{m}$

Ultrasound-modulated optical tomography is a new area of research for the imaging of scattering media, such as biological tissues. In this technology an ultrasonic wave is focused into a scattering medium to modulate light inside the medium. The advantage of this technology is its combination of optical contrast, which can reveal physiological information about biological tissues, and ultrasonic resolution, which is better than the optical resolution achievable by purely optical tomography in thick biological tissues. Marks et al. investigated the modulation of light in homogeneous scattering media with pulsed ultrasound [1]. Wang et al. developed ultrasound-modulated optical tomography in scattering media with continuous-wave ultrasound [2]. Kempe et al. showed experimentally the transition of ultrasonic modulation from the ballistic to the scattered regimes [3]. Wang and $\mathrm{Ku}$ developed a frequency-swept technique to obtain scalable imaging resolution along the acoustic axis by frequency encoding of the light along the acoustic axis [4]. Leveque et al. employed parallel detection of multiple speckles to improve the signal-to-noise ratio, which was demonstrated with one-dimensional images of biological tissues [5]. Yao and Wang further demonstrated this parallel-detection scheme with two-dimensional images of biological tissue [6]. In addition, by combining the parallel-detection and the frequency-swept techniques, Yao et al. obtained two-dimensional images of biological tissues, in which one of the dimensions was along the acoustic axis [7]. In place of transmission configurations, Lev et al. developed a reflection configuration for ultrasoundmodulated optical tomography [8].

Three possible mechanisms have been identified for the ultrasonic modulation of light in scattering media; which mechanism is dominant has, however, remained a mystery. The first mechanism is based on ultrasound-induced variations of the optical properties of the media. As an ultrasonic wave propagates in a scattering medium, the medium is compressed and rarified depending on location and time. Variations of density cause the optical properties of the medium-including the absorption coefficient, the scattering coefficient, and the index of refraction - to vary. Accordingly, the detected intensity of light varies with the ultrasonic wave. This mechanism, recently modeled by Mahan et al. [9], does not require the use of a coherent light source. However, ultrasonic modulation of incoherent light has not been experimentally observed because it is too weak.

The second mechanism is based on variations of the optical phase in response to ultrasound-induced displacements of scatterers. The displacements of scatterers, assumed to follow ultrasonic amplitudes, modulate the physical path lengths of light traversing through the ultrasonic field. Multiply scattered light accumulates modulated physical path lengths along its path. Consequently, the intensity of the speckles formed by the multiply scattered light fluctuates with the ultrasonic wave. A theory given by Leutz and Maret [10] modeled this mechanism but is valid only when the scattering mean free path is much greater than the acoustic wavelength.

The third mechanism is based on variations of the optical phase in response to ultrasonic modulation of the index of refraction. As a result of ultrasonic modulation of the index of refraction, the optical phase between two consecutive scattering events is modulated. Multiply scattered light accumulates modulated phases along its path. As in the second mechanism, the modulated phase causes the intensity of the speckles formed by the multiply scattered light to vary with the ultrasonic wave. This mechanism has never been modeled. Both the second and third mechanisms require the use of coherent light.

In this Letter, I present an analytic model based for the first time on both of the mechanisms for the ultrasonic modulation of coherent light. Further, I compare the relative contributions from the two mechanisms and verify the analytic model with an independent Monte Carlo model.

A plane ultrasonic wave is assumed to irradiate uniformly a homogeneous scattering medium. The autocorrelation function of the electric field, $E(t)$, of the scattered 
light can be expressed as

$$
G_{1}(\tau)=\int_{0}^{\infty} p(s)\left\langle E_{s}(t) E_{s}^{*}(t+\tau)\right\rangle d s,
$$

where the averaging is over time $t, E_{s}$ is the electric field of the scattered light of path length $s$, and $p(s)$ is the probability density function of $s$. The contributions to the autocorrelation function from Brownian motion and from the ultrasonic field are independent and can be treated separately. For conciseness, only the ultrasonic contribution is considered here.

The scattering medium, with a mean free path $l$, consists of noninteracting spherical Rayleigh scatterers. The ultrasonic contribution to the autocorrelation function by $s / l$ scattering events along path $s$ is

$$
\left\langle E_{s}(t) E_{s}^{*}(t+\tau)\right\rangle=\left\langle\exp \left\{-i\left[\sum_{j=1}^{s / l+1} \Delta \phi_{n j}(t, \tau)+\sum_{j=1}^{s / l} \Delta \phi_{d j}(t, \tau)\right]\right\}\right\rangle,
$$

where $\Delta \phi_{n j}(t, \tau)=\phi_{n j}(t+\tau)-\phi_{n j}(t), \phi_{n j}$ is the phase variation induced by the modulated index of refraction along the $j$ th free path, $\Delta \phi_{d j}(t, \tau)=\phi_{d j}(t+\tau)-\phi_{d j}(t)$, and $\phi_{d j}$ is the phase variation induced by the modulated displacement of the $j$ th scatterer following the $j$ th free path. When the phase variations are much less than unity, the following approximation holds:

$$
\left\langle E_{s}(t) E_{s}^{*}(t+\tau)\right\rangle=\exp \left\{-\frac{1}{2}\left\langle\left[\sum_{j=1}^{s / l+1} \Delta \phi_{n j}(t, \tau)+\sum_{j=1}^{s / l} \Delta \phi_{d j}(t, \tau)\right]^{2}\right\rangle\right\} .
$$

On the one hand, the phase variation from the $j$ th free path is

$$
\phi_{n j}(t)=\int_{0}^{l_{j}} k_{0} \Delta n\left(\mathbf{r}_{j-1}, s_{j}, \theta_{j}, t\right) d s_{j},
$$

where $l_{j}$ is the length of the $j$ th free path, $k_{0}$ is the optical wave vector in vacuo, $\Delta n$ is the modulated index of refraction, $\mathbf{r}_{j}$ is the location of the $j$ th scatterer, $s_{j}$ is the distance along the $j$ th free path, and $\theta_{j}$ is the angle between the optical wave vector of the $j$ th free path and the acoustic wave vector $\mathbf{k}_{a}$.

The modulated index of refraction is

$$
\begin{array}{r}
\Delta n\left(\mathbf{r}_{j-1}, s_{j}, \theta, t\right)=n_{0} \eta k_{a} A \sin \left(\mathbf{k}_{a} \cdot \mathbf{r}_{j-1}+k_{a} s_{j}\right. \\
\left.\times \cos \theta_{j}-\omega_{a} t\right),
\end{array}
$$

where $n_{0}$ is the background index of refraction, $k_{a}$ is the amplitude of $\mathbf{k}_{a}, A$ is the acoustic amplitude, $\omega_{a}$ is the acoustic angular frequency, and $\eta$ is related to the adiabatic piezooptical coefficient of the material $\partial n / \partial p$, the density $\rho$, and the acoustic velocity $\nu_{a}: \eta=(\partial n / \partial p) \rho \nu_{a}^{2}$.

By completing the integration in Eq. (4), one obtains

$$
\begin{aligned}
\phi_{n j}(t)= & 2 n_{0} k_{0} \eta A \sin \left(\mathbf{k}_{a} \cdot \mathbf{r}_{j-1}+k_{a} l_{j} \cos \theta_{j} / 2-\omega_{a} t\right) \\
& \times \sin \left(k_{a} l_{j} \cos \theta_{j} / 2\right) / \cos \theta_{j} .
\end{aligned}
$$

The following variance of phase variation can be expanded into quadratic and cross terms:

$$
\left\langle\left[\sum_{j=1}^{s / l+1} \Delta \phi_{n j}(t, \tau)\right]^{2}\right\rangle=\left(\frac{s}{l}+1\right)\left\langle\left[\Delta \phi_{n j}(t, \tau)\right]^{2}\right\rangle+2 \sum_{j=2}^{s / l+1} \sum_{k=1}^{j-1}\left\langle\Delta \phi_{n j}(t, \tau) \Delta \phi_{n k}(t, \tau)\right\rangle .
$$

Each quadratic-term average in Eq. (7) is

$$
\begin{aligned}
\left\langle\left[\Delta \phi_{n j}(t, \tau)\right]^{2}\right\rangle= & \left(4 n_{0} k_{0} \eta A\right)^{2} \sin ^{2}\left(\omega_{a} \tau / 2\right)\left\langle\cos ^{2}\left(\mathbf{k}_{a} \cdot \mathbf{r}_{j-1}+k_{a} l_{j} \cos \theta_{j} / 2-\omega_{a} t-\omega_{a} \tau / 2\right)\right\rangle \\
& \times\left\langle\sin ^{2}\left(k_{a} l_{j} \cos \theta_{j} / 2\right) / \cos ^{2} \theta_{j}\right\rangle .
\end{aligned}
$$

The first average over time $t$ yields $1 / 2$, and the second average over $l_{j}$ and $\cos \theta_{j}$ with probability density functions $p\left(l_{j}\right)=\exp \left(-l_{j} / l\right) / l$ and $p\left(\cos \theta_{j}\right)=1 / 2$ equals

$$
\alpha_{n 1}=k_{a} l \tan ^{-1}\left(k_{a} l\right) / 2 .
$$

Each cross-term average in Eq. (7) is

$$
\begin{aligned}
\left\langle\Delta \phi_{n j}(t, \tau) \Delta \phi_{n k}(t, \tau)\right\rangle= & \left(4 n_{0} k_{0} \eta A\right)^{2} \sin ^{2}\left(\omega_{a} \tau / 2\right)\left\langle(1 / 2) \sin \left(k_{a} l_{j} \cos \theta_{j}\right) / \cos \theta_{j}\right\rangle \\
& \times\left\langle(1 / 2) \sin \left(k_{a} l_{k} \cos \theta_{k}\right) / \cos \theta_{k}\right\rangle \prod_{m=k+1}^{j-1}\left\langle\cos \left(k_{a} l_{m} \cos \theta_{m}\right)\right\rangle .
\end{aligned}
$$

Each of the first two averages in Eq. (10) equals $\beta=\pi / 4-1 /\left(2 k_{a} l\right)\left\{{ }_{2} F_{1}\left[1,1 / 2,3 / 2,-1 /\left(k_{a} l\right)^{2}\right]\right\}$, where ${ }_{2} F_{1}[]$ is the generalized hypergeometric function [11], and the third average equals $\chi=\tan ^{-1}\left(k_{a} l\right) /\left(k_{a} l\right)$. 
With some algebraic operations, Eq. (7) becomes

$$
\begin{aligned}
\left\langle\left[\sum_{j=1}^{s / l+1} \Delta \phi_{n j}(t, \tau)\right]^{2}\right\rangle= & \left(\frac{s}{l}+1\right)\left(2 n_{0} k_{0} A\right)^{2} \delta_{n} \\
& \times\left[1-\cos \left(\omega_{a} \tau\right)\right],
\end{aligned}
$$

where $\delta_{n}=\left(\alpha_{n 1}+\alpha_{n 2}\right) \eta^{2}$, in which

$$
\alpha_{n 2}=2 \beta^{2} /(1-\chi)
$$

is the contribution from the cross terms in the diffusion limit $(s / l \gg 1)$.

On the other hand, the phase variation from the $j$ th scattering event is

$$
\phi_{d j}(t)=-n_{0} k_{0}\left(\hat{\mathbf{k}}_{j+1}-\hat{\mathbf{k}}_{j}\right) \cdot \mathbf{A} \sin \left(\mathbf{k}_{a} \cdot \mathbf{r}_{j}-\omega_{a} t\right),
$$

where $\hat{\mathbf{k}}_{j}$ is the unit optical wave vector for the $j$ th free path, and $\mathbf{A}$ is the acoustic-amplitude vector. The follow- ing variance of phase variation can be derived similarly to the one for Eq. (11):

$$
\left\langle\left[\sum_{j=1}^{s / l} \Delta \phi_{d j}(t, \tau)\right]^{2}\right\rangle=\frac{s}{l}\left(2 n_{0} k_{0} A\right)^{2} \delta_{d}\left[1-\cos \left(\omega_{a} \tau\right)\right]
$$

where $\delta_{d}=1 / 6$, and the following relation for Rayleigh scattering is used in the derivation: $\left\langle\left[\left(\hat{\mathbf{k}}_{j+1}-\right.\right.\right.$ $\left.\left.\left.\hat{\mathbf{k}}_{j}\right) \cdot \mathbf{A}\right]^{2}\right\rangle=(1 / 3)\left\langle\left(\hat{\mathbf{k}}_{j+1}-\hat{\mathbf{k}}_{j}\right)^{2}\right\rangle A^{2}=2 A^{2} / 3$. An important difference between $\delta_{d}$ and $\delta_{n}$ is that the average sum of the cross terms among the phase variations $\Delta \phi_{d j}$ in Eq. (14) vanishes in the diffusion limit. In other words, the contributions from displacement by different scattering events are independent; in contrast, the contributions from the index of refraction by different free paths are coupled.

The autocorrelation function in Eq. (1) becomes

$$
G_{1}(\tau)=\int_{0}^{\infty} p(s) \exp \left\{-(2 s / l)\left(\delta_{n}+\delta_{d}\right)\left(n_{0} k_{0} A\right)^{2}\left[1-\cos \left(\omega_{a} \tau\right)\right]\right\} d s
$$

in the diffusion limit, where the independence between $\Delta \phi_{n j}$ and $\Delta \phi_{d k}$ is used. A coherent optical beam from an extended plane source is assumed to be incident upon a slab of thickness $L$, and the transmitted light is detected from a point. The function $p(s)$ is given by diffusion theory with a zero-boundary condition [12]. By carrying out the integration in Eq. (15) [13], one obtains

$$
G_{1}(\tau)=\frac{(L / l) \sinh \left[\left\{\varepsilon\left[1-\cos \left(\omega_{a} \tau\right)\right]\right\}^{1 / 2}\right]}{\sinh \left[(L / l)\left\{\varepsilon\left[1-\cos \left(\omega_{a} \tau\right)\right]\right\}^{1 / 2}\right]}
$$

where $\varepsilon=6\left(\delta_{n}+\delta_{d}\right)\left(n_{0} k_{0} A\right)^{2}$.

The intensity of the modulated speckles at frequency $n \omega_{a}$ can be calculated based on the Wiener-Khinchin theorem: $I_{n}=\int_{0}^{T_{a}} \cos \left(n \omega_{a} \tau\right) G_{1}(\tau) d \tau / T_{a}$, where $T_{a}$ is the acoustic period. The modulation depth, $M$, is defined as the ratio between the intensity at the fundamental frequency $I_{1}$ and the unmodulated intensity $I_{0}$.

Figure 1 plots the ratio between $\delta_{n}$ and $\delta_{d}$ as a function of $k_{a} l$. It can be seen from the above derivation that $\delta_{n}$ and $\delta_{d}$ are related to the average contributions per free path and per scattering event, respectively, to the ultrasonic modulation of light intensity. The contribution from the index of refraction $\delta_{n}$ increases with $k_{a} l$ because a longer free path relative to the acoustic wavelength accumulates a greater phase modulation. By contrast, the contribution from displacement $\delta_{d}$ stays constant at $1 / 6$, independent of $k_{a}$ and $l$. As a result, the ratio between $\delta_{n}$ and $\delta_{d}$ increases with $k_{a} l$ and crosses a critical point at $k_{a} l=0.559$ or $l=0.0890 \lambda_{a}$, where the ratio is unity. When $k_{a} l$ is less than this critical point, the contribution from the index of refraction is slightly less than the contribution from displacement; beyond this critical point, the contribution from the index of refraction increases and can significantly outmatch the contribution from displacement. It is worth noting that, although the ratio between $\delta_{n}$ and $\delta_{d}$ depends on $\eta$ and $k_{a} l$, it is independent of $n_{0}, k_{0}, l, A$, and $L$.

The contribution from the index of refraction has two components (Fig. 2): one is from the individual free paths, as determined by $\alpha_{n 1}$, and the other is from the cross products among the free paths, as determined by $\alpha_{n 2}$. The contribution from the individual free paths $\alpha_{n 1}$ increases with $k_{a} l$ for the same reason stated previously - a longer free path relative to the acoustic wavelength accumulates a greater phase modulation. However, the contribution from the cross products among the free paths $\alpha_{n 2}$ decreases only slightly with an increasing $k_{a} l$ as a result of two counteracting effects - increasing phase modulation from each free path as determined by $\beta^{2}$ in Eq. (12) and decreasing coupling among the free paths as determined by $1 /(1-\chi)$ in Eq. (12). The ratio between $\alpha_{n 1}$ and $\alpha_{n 2}$ increases with $k_{a} l$ and crosses a critical point at $k_{a} l=2.33$ or $l=0.371 \lambda_{a}$, where the two contributions are equal; however, this ratio is independent of $\eta$ as well as $n_{0}, k_{0}$, $l, A$, and $L$.

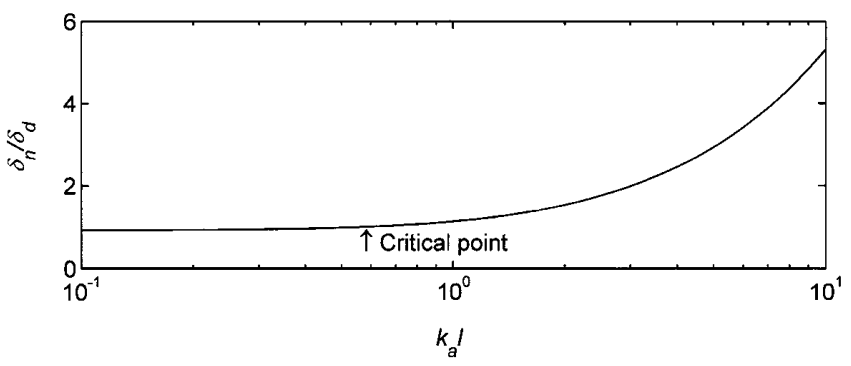

FIG. 1. The ratio between $\delta_{n}$ and $\delta_{d}$ as a function of $k_{a} l$. The following parameters for water are used to calculate $\eta$ : $\partial n / \partial p=1.466 \times 10^{-10} \mathrm{~m}^{2} / \mathrm{N}, \rho=1000 \mathrm{~kg} / \mathrm{m}^{3}$, and $\nu_{a}=$ $1480 \mathrm{~m} / \mathrm{s}$. 

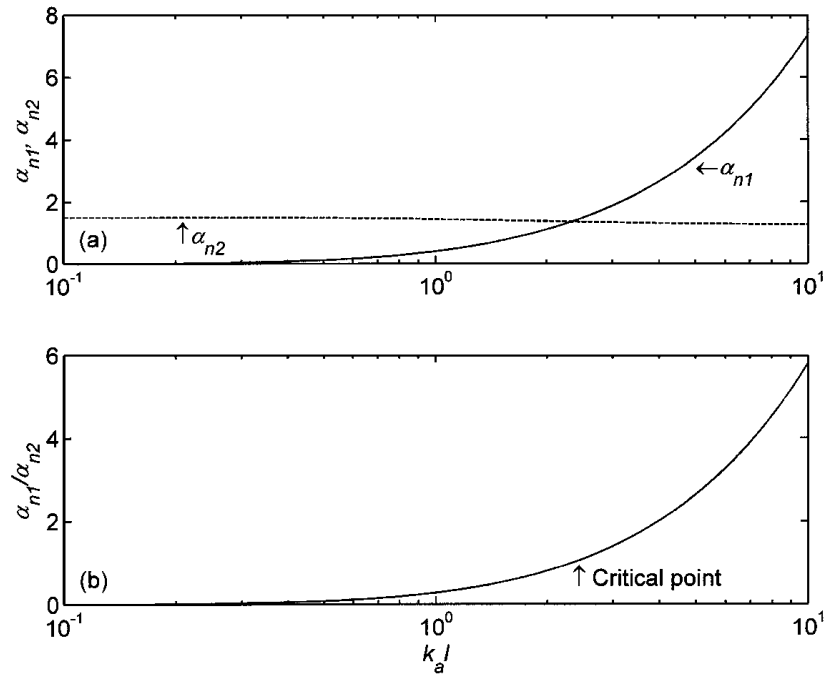

FIG. 2. (a) The two components of modulation from the index of refraction, $\alpha_{n 1}$ and $\alpha_{n 2}$, and (b) their ratio $\alpha_{n 1} / \alpha_{n 2}$ as a function of $k_{a} l$.

Figure 3 shows the modulation depth as a function $k_{a} l$ with a constant $k_{a}$ contributed from the index of refraction alone, $M_{n}$, the modulation depth contributed from displacement alone, $M_{d}$, and the modulation depth contributed from both, $M_{\text {sum }}$. The symbols are from an independent Monte Carlo simulation accomplished by programming Eqs. (1) and (2) into a public-domain software package for light transport in scattering media [14]. The analytic model presented here agrees with the Monte Carlo model very well. The modulation depths decrease with $k_{a} l$ because the average number of free paths or scattering events, related to $L / l$, decreases with an increasing $l$ and its effect outweighs the increasing modulation from each free path. A plot of the ratio between $M_{n}$ and $M_{d}$ (not shown) resembles Fig. 1 because the modulation depth $M$ is proportional to $\varepsilon$ for weak modulations, as can be shown from Eq. (16).

In summary, an analytic model of the ultrasonic modulation of multiply scattered coherent light in scattering media is provided. The model is based on two mechanisms: the ultrasonic modulation of the index of refraction, which causes a modulation of the optical path lengths between consecutive scattering events, and the ultrasonic modulation of the displacements of Rayleigh scatterers, which causes a modulation of optical path lengths upon each scattering event. Multiply scattered light accumulates modulated optical path lengths along its path. Consequently, the intensity of the speckles that are formed by the multiply scattered light is modulated. In water solutions, for example, the contribution from the index of refraction is slightly less than the contribution from displacement when the scattering mean free path is less than a critical fraction (0.0890) of the acoustic wavelength, and it becomes increasingly greater than the contribution from displace-

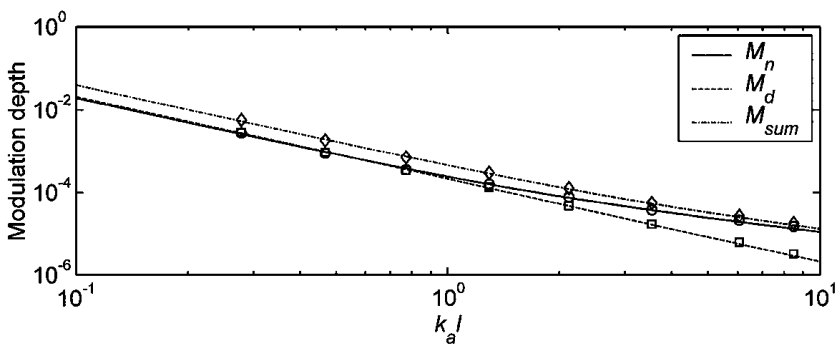

FIG. 3. The modulation depths $M_{n}, M_{d}$, and $M_{\text {sum }}$ as a function $k_{a} l$ with a constant $k_{a}$. Besides the parameters for Fig. 1, the following parameters are used: $\omega_{a}=2 \pi \mathrm{MHz}, n_{0}=1.33$, $\lambda_{0}=500 \mathrm{~nm}, L=5 \mathrm{~cm}$, and $A=0.01 \mathrm{~nm}$.

ment beyond this critical point. This analytic model agrees well with an independent Monte Carlo model. Both of the mechanisms are coherent phenomena, requiring the use of a coherent light source. The extension of the present theory to non-Rayleigh scatterers will be presented elsewhere [15]. This work clarifies the mystery about the mechanisms of ultrasonic modulation of light in scattering media.

This project was sponsored in part by National Science Foundation Grant No. BES-9734491, Texas Higher Education Coordinating Board Grant No. 000512-01231999, and National Institutes of Health Grants No. R29 CA68562 and R01 CA71980.

*Electronic address: LWang@tamu.edu

[1] F. A. Marks, H. W. Tomlinson, and G. W. Brooksby, Proc. Soc. Photo-Opt. Instrum. Eng. 1888, 500 (1993).

[2] L.-H. Wang, S. L. Jacques, and X. Zhao, Opt. Lett. 20, 629 (1995).

[3] M. Kempe, M. Larionov, D. Zaslavsky, and A. Z. Genack, J. Opt. Soc. Am. 14, 1151 (1997).

[4] L.-H. V. Wang and G. Ku, Opt. Lett. 23, 975 (1998).

[5] S. Leveque, A. C. Boccara, M. Lebec, and H. Saint-Jalmes, Opt. Lett. 24, 181 (1999).

[6] G. Yao and L.-H. V. Wang, Appl. Opt. 39, 659 (2000).

[7] G. Yao, S.-L. Jiao, and L.-H. V. Wang, Opt. Lett. 25, 734 (2000).

[8] A. Lev, Z. Kotler, and B. G. Sfez, Opt. Lett. 25, 378 (2000).

[9] G. D. Mahan, W. E. Engler, J. J. Tiemann, and E. Uzgiris, Proc. Natl. Acad. Sci. U.S.A. 95, 14015 (1998).

[10] W. Leutz and G. Maret, Physica (Amsterdam) 204B, 14 (1995).

[11] G. B. Arfken and H.J. Weber, Mathematical Methods for Physicists (Academic, San Diego, CA, 1995), 4th ed.

[12] M.S. Patterson, B. Chance, and B. C. Wilson, Appl. Opt. 28, 2331 (1989).

[13] D. J. Pine, D. A. Weitz, P. M. Chaikin, and E. Herbolzheimer, Phys. Rev. Lett. 60, 1134 (1988).

[14] L.-H. Wang, S. L. Jacques, and L.-Q. Zheng, Comput. Methods Prog. Biomed. 47, 131 (1995). Software available at http://oilab.tamu.edu.

[15] L.-H. V. Wang, Opt. Lett. (to be published). 